\title{
Reflux oesophagitis secondary to duodenal diaphragm in an adult
}

\author{
GEORGEW. JOHNSTON AND H. MORRIS STEVENSON \\ From the Royal Victoria Hospital, Belfast
}

Congenital duodenal stenosis or diaphragm usually presents as a neonatal obstruction requiring urgent surgery, but there are rare instances of survival into adult life without treatment and sometimes with surprisingly few symptoms. Hudson (1961), in a review of the literature, found only 11 such cases and added one of his own. In the same year, Threadgill and Hagelstein (1961) reported two further cases in men aged 23 and 45 years. Flachs (1962) reported on four patients with incomplete septa of the duodenal bulb diagnosed radiologically, but in only one case was the septum demonstrated anatomically. Recently, Ross, Isaac, and Lyon (1964) reported the necropsy findings in a 66-year-old man with a persistent diaphragm at the junction of the first and second parts of the duodenum. Vomiting and weight loss have been prominent features in the cases previously reported, but we now report a patient who presented with dysphagia.

\section{CASE REPORT}

D. M., aged 19 years, first came to hospital in February 1964 with a four-month history of waterbrash, heartburn, and difficulty in swallowing solid food. Barium studies revealed narrowing of the oesophagus at the level of the left bronchus (Fig. 1), and screening demonstrated free gastro-oesophageal reflux. There was gross dilatation of the stomach and proximal duodenum due to hold-up at the beginning of the third part of the duodenum (Fig. 2). The 24-hour films showed that most of the barium remained in the stomach and proximal duodenum. On oesophagoscopy there was reflux of bile-stained gastric contents, and a zone of oesophagitis with flaking of the mucosa at $25 \mathrm{~cm}$. from the upper incisor teeth. A biopsy of the area showed inflammation and ulceration of the oesophageal mucosa but no evidence of ectopic gastric mucosa.

At laparotomy in April 1964 there was no evidence of hiatal hernia or gastric or duodenal ulceration. The first and second parts of the duodenum were distended and rotated to the left, and the walls of both the stomach and proximal duodenum were thickened. Using Kocher's manœuvre, the duodenum was mobilized and was found to assume normal calibre beyond the junction of its second and third parts. Duodenotomy, just proximal to the narrow segment, revealed a diaphragm with a $3 \mathrm{~mm}$. opening eccentrically placed, close to the postero-medial wall. The diaphragm was excised, and a retrocolic duodenojejunal anastomosis was performed with the stoma centred on the site of the excised web. Histology of the diaphragm showed only normal duodenal mucosa without ulceration or inflammation.

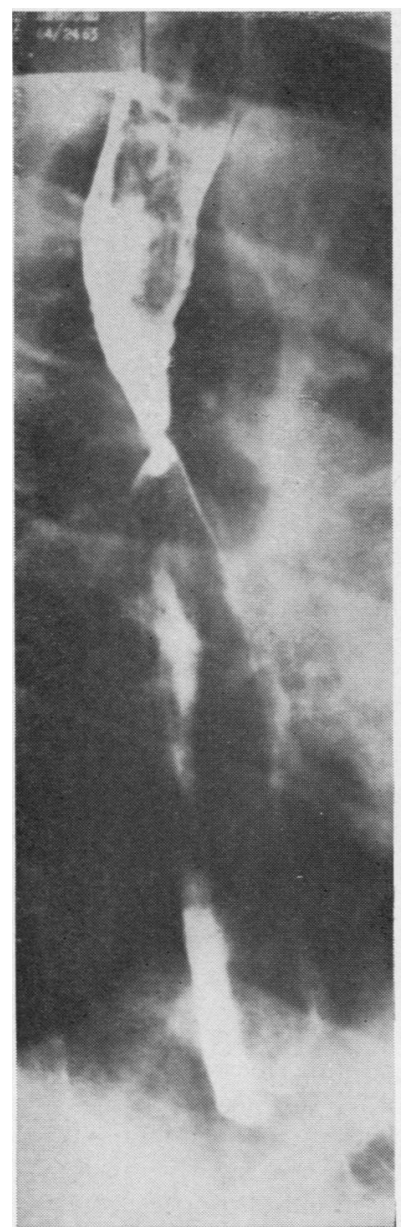

FIG. 1. Barium swallow showing site of narrowing at the level of the left bronchus. 


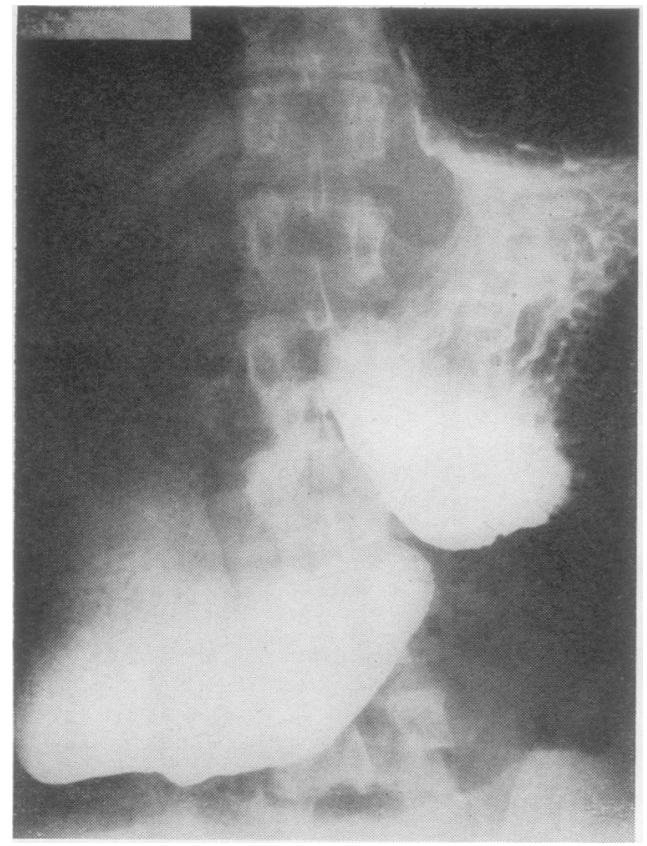

FIG. 2. Barium meal showing massive dilatation of the first and second parts of the duodenum.

Since operation the patient has been free of the symptoms of reflux, and his difficulty in swallowing has resolved completely. On repeat barium meal examination in September 1964, the oesophagus dilated easily in the region of the previous narrowing, refiux was absent, and there was no hold-up in either the stomach or duodenum. Oesophagoscopy in September 1964 showed no evidence of obstruction, oesophagitis, or reflux.

\section{DISCUSSION}

As Maclaurin (1963) has shown, oesophageal reflux is prevented in part in the normal subject by the action of the intra-abdominal pressure on the lower intra-abdominal segment of the oesophagus. This is reinforced by the mucosal seal at the cardia, which is maintained, at least partially, by the contraction of the internal sphincter. Reflux may result where the intragastric pressure becomes higher than the intra-abdominal pressure, or where the intra-abdominal part of the oesophagus is displaced into the chest. As far back as 1906, Tileston pointed out the possible significance of pyloric obstruction in producing dilatation of the stomach and thereby relative insufficiency of the cardia. In the present case, the rise in intragastric pressure was due to duodenal obstruction, and reflux ceased when the obstructing diaphragm was removed. Since the diaphragm was situated distal to the ampulla of Vater, the regurgitant fluid contained bile and trypsin, giving rise to severe tryptic oesophagitis, as sometimes occurs after total or even partial gastrectomy (Bingham, 1958 ; McKeown, 1958 ; Kennedy, 1959 ; Cox, 1961).

Although a persistent duodenal diaphragm may occur anywhere in the duodenum, it is most commonly situated near the ampulla of Vater (Aird, 1957 ; Hudson, 1961). Some consider duodenojejunostomy the treatment of choice for this condition (Maingot, 1961 ; Harkins and Nyhus, 1962), and this is undoubtedly the best procedure in the infant in whom there has been no long-standing dilatation of the duodenum. In adults, however, duodeno-jejunostomy alone rarely relieves the preoperative symptoms, and this had led Threadgill and Hagelstein (1961) to recommend Billroth II gastrectomy as the operation of choice. Hudson (1961) considers duodenotomy with excision of the web sufficient, but if the web lies near the ampulla this can be a hazardous procedure. It is well to remember that the anatomy can be grossly distorted by the distension of the proximal duodenum, and it is therefore important to identify the duodenal papilla before excising the web. In our patient the combination of excision of the diaphragm and duodeno-jejunostomy has relieved the obstruction, stopped the reflux, and allowed the oesophagitis to heal.

\section{SUMMARY}

A case of reflux oesophagitis, probably tryptic in origin, secondary to obstruction by a congenital duodenal diaphragm, is reported in a 19-year-old man. Excision of the diaphragm and duodenojejunal anastomosis has given a satisfactory result.

\section{REFERENCES}

Aird, I. (1957). A Companion in Surgical Studies, 2nd ed., Livingstone, Edinburgh.

Bingham, J. A. W. (1958). Oesophageal structures aft er gastric surgery and naso-gastric intubation. Brit. med. J., 2, 817 .

Cox, K. R. (1961). Oesophageal stricture after partial gastrectomy. Brit. J. Surg., 49, 307.

Flachs, K. (1962). Incomplete congenital septa of the duodenal bulb. Acta radiol. (Stockh.), 57, 237.

Harkins, H. N., and Nyhus, L. M. (1962). Surgery of the Stomach and Duodenum, 1st ed., Churchill, London.

Hudson, C. N. (1961). Congenital diaphragm of the duod enum causing intestinal obstruction in an adult. Brit. J. Surg., 49, 234.

Kennedy, T. (1959). Total gastrectomy. Postgrad. med. $J_{\text {., 35, }} 138$.

McKeown, K. C. (1958). Oesophageal stenosis after partial gastrectomy. Brit. med. J., 2, 819.

Maclaurin, C. (1963). The intrinsic sphincter in the prevention of gastro-oesophageal reflux. Lancet, 2, 801.

Maingot, R. (1961). Abdominal Operations, 4th ed., Lewis, London.

Ross, J. A., Isaac, A. R., and Lyon, D. S. (1964). Duodenal diaphragm presenting in late adult life. Brit. J. Surg., 51, 632.

Threadgill, F. D., and Hagelstein, A. (1961). Duodenal diaphragm in the adult. Arch. Surg., 83, 878.

Tileston, W. (1906). Peptic ulcer of the oesophagus. Amer. J. med. $S c i, 132,240$. 